\section{Estudo da técnica de venografia dos dígitos de vacas}

[Study of Venography technique in digits of cows]

M.G. Loureiro ${ }^{1}$, J.R.B. Silva ${ }^{2 *}$, M. Rodrigues ${ }^{3}$, L.A. Rafael ${ }^{4}$, L.C. Vulcano ${ }^{2}$, C.A. Hussni $^{2}$, A.L.G. Alves ${ }^{2}$, M.J. Watanabe ${ }^{2}$, C.A. Rodrigues ${ }^{2}$

${ }^{1}$ Instituto Federal Catarinense - Concórdia, SC

${ }^{2}$ Universidade Estadual Paulista, UNESP - FMVZ - Botucatu, SP

${ }^{3}$ Universidade do Oeste Paulista - Presidente Prudente, SP

${ }^{4}$ Universidade Federal Pelotas - Pelotas, RS
M.G. Loureiro1

https://orcid.org/0000-0002-9671-7633

J.R.B. Silva2*

https://orcid.org/0000-0002-5027-2384 M. Rodrigues3

https://orcid.org/0000-0002-0429-9375 L.A. Rafael4

https://orcid.org/0000-0002-7282-0847

L.C. Vulcano2

https://orcid.org/0000-0001-8307-5187 C.A. Hussni2

https://orcid.org/0000-0001-5421-2904 A.L.G. Alves2

http://orcid.org/0000-0001-9092-7819 M.J. Watanabe2

https://orcid.org/0000-0002-7317-4250 C.A. Rodrigues 2

https://orcid.org/0000-0002-4837-463X

\title{
RESUMO
}

O objetivo deste estudo foi descrever a técnica de venografia retrógrada podal em vacas, comparando os acessos da veia digital dorsal comum III com a digital comum II ou IV, nos membros torácicos e pélvicos, mediante a administração de dois diferentes volumes de contraste. Foram utilizados 53 membros torácicos e pélvicos de 14 vacas, com o torniquete de borracha posicionado a $5 \mathrm{~cm}$ proximal aos paradígitos. Administraram-se 10mL do diatrizoato de meglumina em 24 membros (grupo 1), sendo 13 na veia digital dorsal comum III pelo acesso 1 (A1) e 11 na digital II ou IV no acesso 2 (A2). No grupo 2, administraram-se $20 \mathrm{~mL}$ em 29 membros, sendo 15 pelo A1 e 19 pelo A2. Após a administração do contraste, as radiografias foram repetidas a cada 20 segundos até 120 segundos. $\mathrm{O}$ grau de preenchimento vascular foi maior no grupo 2, não diferindo entre membros e acessos venosos. Conclui-se que a administração de $20 \mathrm{~mL}$ de contraste apresentou melhor preenchimento vascular e radiopacidade, não havendo diferença entre 20 e 120 segundos após a administração do contraste na qualidade radiográfica, independentemente do acesso venoso.

Palavras-chave: contraste, laminite, podologia, raios $\mathrm{X}$, venograma

\begin{abstract}
The aim of this study was to describe the technique of retrograde venography foot in cows, comparing the approaches of the dorsal common digital vein III with the digital commons II or IV, thoracic and pelvic by administering two different volumes of contrast members. Fifty three fore and hindlimbs of 14 cows were used, a rubber tourniquet was placed at $5 \mathrm{~cm}$ above accessory digit. Diatrizoatemeglumine was administered at 10mL to 24 members (group 1), 13 dorsal common digital vein III for access 1 (A1), and 11 digital II or IV access 2 (A2). In group 2 20mL was administered to 29 members, 15 by 19 in A1 and A2. After contrast administration, the radiographs were repeated every 20 seconds until 120 seconds. The degree of vascular filling was greater in group 2, independent of venous access, member or moment. There was no significant difference in the degree of radiopacity of radiographic images when compared to the venous access, time and a member of both groups. We conclude that administration of $20 \mathrm{~mL}$ of contrast showed better vascular filling and radiopacity, with no difference between 20 and 120 seconds after contrast administration in independent radiographic quality venous access.
\end{abstract}

Keywords: contrast, laminitis, podiatry, x rays, venogram

\section{INTRODUÇÃO}

Diversos métodos in vivo e in vitro têm sido utilizados para avaliar o fluxo sanguíneo digital. Entre esses métodos estão a angiografia da artéria digital (Akerman et al., 1975) e a

Recebido em 19 de junho de 2017

Aceito em 31 de agosto de 2018

*Autor para correspondência (corresponding author)

E-mail: celso.a.rodrigues@unesp.br venografia (D’Arpe e Bernardini, 2010). O exame venográfico consiste em uma ferramenta diagnóstica para a identificação radiográfica da vascularização venosa, podendo ser realizado no dígito após a administração de um contraste líquido radiopaco em uma veia digital palmar ou plantar (D'Arpe e Bernardini, 2010). 
A venografia complementa os achados clínicos, conferindo mais precisão na elaboração do diagnóstico e prognóstico, o que favorece a tomada de decisão na condução clínica do caso e permite a monitorização mais detalhada da evolução da enfermidade (Rucker, 2010). O venograma também pode evidenciar áreas de necrose antes mesmo que estas sejam detectadas clinicamente (Hood, 1999).

$\mathrm{Na}$ avaliação da venografia, o profissional deve estar familiarizado com a anatomia vascular do dígito e adquirir experiência com os diversos estudos em animais sadios e em outros acometidos de enfermidades podais, para que ele tenha capacidade de diferenciar e evitar o surgimento dos artefatos de técnica (Rucker, 2007; Santos et al., 2015a). O objetivo deste estudo foi descrever a técnica de venografia retrógrada podal em vacas, comparando os acessos da veia digital dorsal comum III com a digital comum II ou IV, nos membros torácicos e pélvicos, mediante a administração de dois volumes diferentes de contraste.

\section{MATERIAL E MÉTODOS}

Foram utilizados 14 bovinos da raça Holandesa, fêmeas, adultas, com peso médio de $610 \mathrm{~kg}$, sem histórico de lesões podais. A ausência de lesões podais foi estabelecida mediante atribuição do escore de locomoção e exame clínico específico (inspeção e palpação das úngulas).

Os animais foram contidos em decúbito lateral direito, em tronco tombador, para casqueamento modelo hidráulico (Horsy Heron ${ }^{\circledR}$, Garça, Brasil), e os membros mantidos em posição apropriada, por meio de cintas de náilon com $150 \mathrm{~cm}$ de comprimento e $5 \mathrm{~cm}$ de largura, fixadas no terço proximal dos metatarsos/metacarpos ao tronco.

Os animais foram divididos, de forma aleatória, em dois grupos. No grupo 1 (G1), composto por seis bovinos, foi utilizado o volume de $10 \mathrm{~mL}$ de diatrizoato de meglumina (Relieve $60 \%{ }^{\circledR}$ ) como contraste e, no grupo 2 (G2), composto por oito bovinos, foram utilizados $20 \mathrm{~mL}$, administrados pela via intravenosa regional sem diluição. Durante a divisão dos dois grupos, como método de randomização, foi realizado o sorteio do animal, sendo o volume contraste administrado de modo semelhante nos quatro membros. Os volumes foram utilizados com base em um estudo piloto com 10 bovinos, fêmeas, adultas, da raça Holandesa, no qual se observou desconforto em todos os animais durante a administração de um volume maior que $25 \mathrm{~mL}$.

Além da divisão dos membros em G1 e G2, estes foram divididos em dois subgrupos. No primeiro, denominado acesso 1 (A1), optou-se pela venopunção da v. digital dorsal comum III, totalizando 28 membros, sendo 13 no G1 e 15 no G2. Nos membros do subgrupo denominado acesso 2 (A2), foi realizada a venopunção na face medial do membro sobre v. digital palmar/plantar comum II ou na face lateral v. digital palmar/plantar comum IV, totalizando 25 membros, sendo 11 no G1 e 14 no G2. Essa variação entre a v. digital comum II ou IV não seguiu nenhum padrão e ocorreu devido à conveniência em se promover uma venopunção adequada e duradoura, minimizando a possibilidade de administração perivascular ou extravasamento do contraste.

Foi realizado o torniquete utilizando-se fita de borracha butílica elástica $(40 \times 3 \times 600 \mathrm{~mm})$ no terço distal do metatarso ou metacarpo, aproximadamente $5 \mathrm{~cm}$ proximal aos paradígitos.

Após tricotomia e antissepsia com aspersão de álcool $70^{\circ}$, foi introduzido um escalpe $19 \mathrm{G}$ no sentido contrário ao fluxo sanguíneo da veia digital, onde se procedeu à colocação do dispositivo $\mathrm{PRN}^{\circledR}$, seguida pela fixação do escalpe sobre o torniquete com esparadrapo. Imediatamente ao término da administração do contraste, os dígitos foram flexionados $\mathrm{e}$ estendidos rápida e levemente, totalizando 10 movimentos sequenciais.

A projeção dorsopalmar $0^{\circ}$ foi utilizada nos membros torácicos e no dorsoplantar $0^{\circ}$ nos membros pélvicos para realização das venografias, sendo repetidas com intervalo de 20 segundos após a administração do contraste até o tempo de 120 segundos, correspondendo aos momentos M1 ao M6.

Os procedimentos radiográficos foram realizados com aparelho emissor de raios $\mathrm{X}$ portátil, com painel digital (DR - Orange 1040HF ${ }^{\circledR}$, Samsung, China), com detector de silicone amorfo e área de $10 "$ x 12", sendo o e-film ${ }^{\circledR}$ o panorama visualizador de imagens utilizado. 
Para a realização das radiografias, a técnica empregada foi $65 \mathrm{kV}$ com $50 \mathrm{mAs}$, mantendo a distância fixa de $60 \mathrm{~cm}$ entre o foco e o filme, com incidência dos raios $\mathrm{X}$ perpendicular ao chassi.

Vale ressaltar que, durante todo o procedimento, os animais permaneceram em decúbito lateral direito. Dessa forma, em nenhum momento durante o exame venográfico ocorreu o apoio dos membros no solo ou em qualquer outra superfície.

Foram estabelecidos dois critérios qualitativos para a avaliação das imagens venográficas, divididos em padrão de preenchimento vascular e grau de radiopacidade do contraste. Os escores de preenchimento instituídos foram: 0: contraste no subcutâneo; 1: presente nas veias da falange média de apenas um dígito; 2: presente nas veias das falanges média e distal de apenas um dos dígitos; 3: presente nas veias das falanges média e distal de um dos dígitos e média do outro; 4: presente nas veias das falanges médias e distal com falha limitada em uma das falanges distais; 5: presente no preenchimento completo das veias das falanges média e distal (Fig. 1).

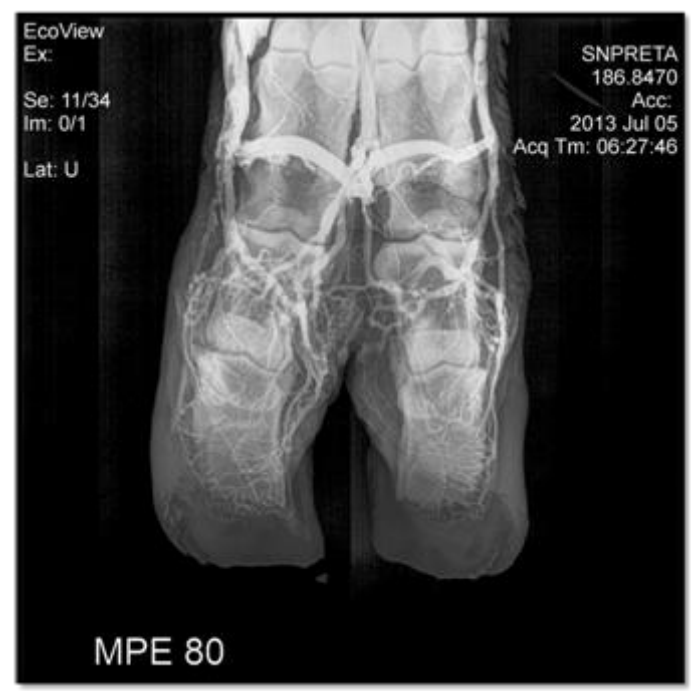

Figura 1. Venografia da região distal a articulação metatarsofalângica do membro pélvico esquerdo de um bovino adulto, em projeção dorsopalmar, do animal $\mathrm{n}^{\circ} 01$, pertencente ao grupo 2 e acesso A2 (M4), em que $100 \%$ dos avaliadores quantificaram em 5 o escore de preenchimento vascular.
No critério grau de radiopacidade, as avaliações foram subjetivas, não sendo fornecido aos avaliadores nenhum padrão para cada escore individualmente. Os escores de radiopacidade foram divididos em: 0 : ausência; 1 : baixa; 2 : média; 3: alta radiopacidade (Fig. 2).

Os arquivos digitais contendo as venografias, tabuladas por animal e membros ao longo do tempo, foram distribuídos entre cinco avaliadores. Estes desconheciam a metodologia experimental, foram selecionados com base em sua competência e apresentavam experiência comprovada na prática clínica e didática em radiologia veterinária.

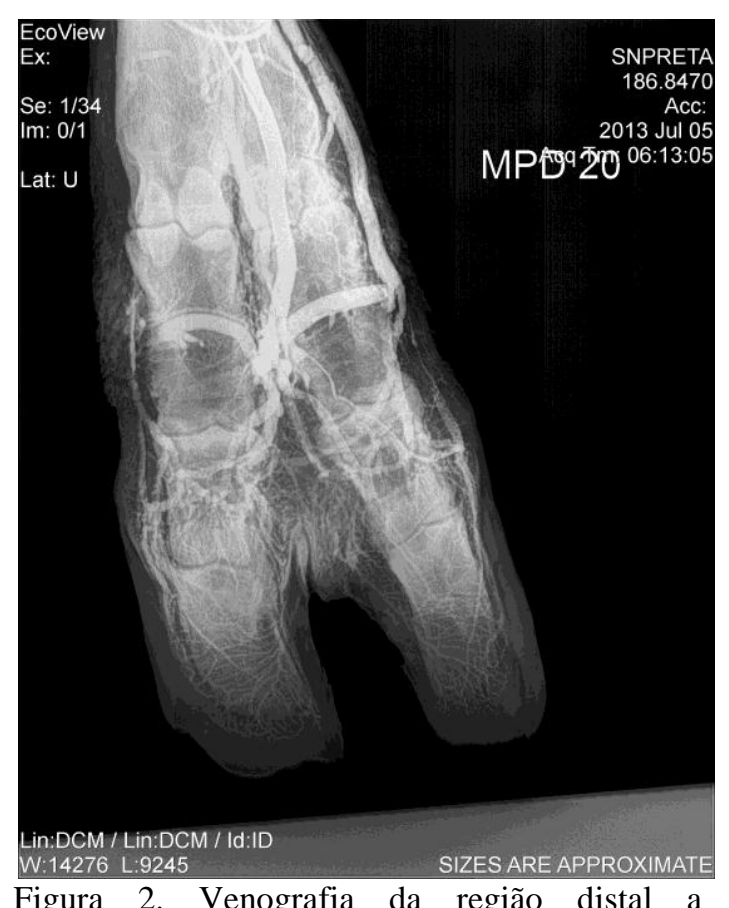

Figura 2. Venografia da região distal a articulação metatarsofalângica do membro pélvico esquerdo de um bovino adulto, em projeção dorsopalmar, do animal $\mathrm{n}^{\circ} 01$, pertencente ao grupo 2 e acesso A1 (M1), em que $100 \%$ dos avaliadores quantificaram em escore 3 para o grau de radiopacidade.

As análises estatísticas dos escores de preenchimento e dos escores de radiopacidade foram realizadas pelos testes de Mann-Whitney, para comparar os grupos segundo os acessos em cada momento; Wilcoxon, para comparar os acessos segundo os grupos em cada momento; Friedman, para comparar os momentos segundo os grupos e os acessos; e teste de Mann-Whitney, 
para comparar os grupos em cada momento $(\mathrm{P}<0,05)$. As análises estatísticas foram efetuadas empregando-se o programa SAS ${ }^{\circledR}$ (2008).

Este trabalho foi aprovado pelo Comitê de Ética da Faculdade de Medicina Veterinária e Zootecnia - FMVZ, Unesp/Botucatu, sob o $\mathrm{n}^{\circ} 131 / 2013$.

\section{RESULTADOS E DISCUSSÃO}

Em virtude do comportamento e das técnicas disponíveis para contenção física na espécie bovina, optou-se pela realização da venografia em decúbito lateral direito em tronco tombador hidráulico, não havendo intercorrências ou dificuldade na realização da técnica, diferentemente da espécie equina, em que esse procedimento é realizado na posição quadrupedal e sob sedação à base de fármacos $\alpha-2$ agonistas (D’Arpe e Bernardini, 2010; Rucker, 2010).

Os volumes preconizados neste estudo não seguem os mesmos padrões recomendados para a espécie equina, em que os volumes podem variar de 25 a $30 \mathrm{~mL}$ para animais entre 450 e $700 \mathrm{~kg}$ (D'Arpe e Bernardini, 2010; Baldwin e Pollitt, 2010), até $36 \mathrm{~mL}$ para equinos adultos de raças grandes (Redden, 2001), sendo recomendado de 25 a $30 \mathrm{~mL}$ para bovinos adultos (Santos et al., 2015b), ao passo que, para ovinos e caprinos,são recomendados 10mL (Santos et al., 2015a). A alteração no volume é explicada pelas observações clínicas de um estudo piloto destes autores, em que se identificou pressão excessiva no êmbolo da seringa e desconforto dos animais durante a administração do contraste após $25 \mathrm{~mL}$ em 10 vacas, independentemente da veia em que foi realizado o acesso. Tendo em vista que a pressão excessiva é determinante na ocorrência de falhas na venografia (Rucker, 2007), optou-se pelo volume máximo de $20 \mathrm{~mL}$ de contraste.

A venopunção digital dorsal comum III foi executada com maior facilidade tanto nos membros torácicos quanto nos pélvicos quando comparada ao acesso da v. digital palmar/plantar comum II e IV. Essa facilidade na venopunção e na manutenção da venóclise pode ser decisiva para a realização de uma venografia adequada, minimizando as falhas da técnica. Considerando a comparação de acessos na espécie equina, tanto a veia quanto a artéria digital dorsal comum III estão ausentes (Budras et al., 2005), assim não foi possível comparar com os dados da literatura entre as duas espécies.

Em estudos recentes, existe a recomendação para a utilização da veia digital medial em equinos, como via para a administração do contraste (Rucker, 2010). Baldwin e Pollitt (2010) relatam que a administração do contraste na veia digital, independentemente de ser lateral ou medial, não influencia na venografia. As análises estatísticas não evidenciaram diferenças significativas nas características radiográficas, independentemente do acesso venoso, corroborando os resultados encontrados na espécie equina (Baldwin e Pollitt, 2010; Rucker, 2010).

Nos membros onde a força da gravidade se opõe ao fluxo sanguíneo, as válvulas permitem o fluxo sanguíneo em direção ao coração, mas não na direção inversa (Moore et al., 2014). Também se relata que a flexão do carpo e o apoio dos membros no solo, após a administração do contraste, favorecem a perfusão de vasos laminares na extremidade do membro (Redden, 2001; Rucker, 2010, D’Arpe e Bernardini, 2010). Nos bovinos deste estudo, somente a flexão manual da extremidade distal dos membros, parece não ter sido suficiente para a abertura das válvulas venosas, colaborando para uma menor ou ausência de difusão do contraste em determinadas regiões do dígito, em diferentes momentos.

O preenchimento inadequado pelo contraste na vasculatura digital dos bovinos estudados deve ser considerado ao se pretender utilizar a técnica de venografia em ruminantes para avaliar processos inflamatórios, como no equino (Ackerman et al., 1975; Baldwin e Pollitt, 2010). As diferenças anatômicas vasculares na extremidade dos membros dessas espécies, especialmente a presença de numerosas válvulas venosas, devem ser o principal responsável pelas falhas de preenchimento observadas nos animais saudáveis (König e Liebich, 2011; Tortora e Derrickson, 2012).

Considerando os valores para o preenchimento vascular pelo contraste (Tab. 1), os valores medianos observados no G2 são maiores quando comparados aos do G1. Ainda nesse aspecto, no G1 não foram observados valores de escore máximos (5) em nenhum momento. 
Ao se compararem os grupos, existe diferença significativa $(\mathrm{P}<0,05)$ relacionada ao escore de preenchimento vascular. Quando se consideram os momentos e os diferentes acessos avaliados, evidencia-se que não existe diferença significativa $(\mathrm{P}>0,05)$ entre estes.

Tabela 1. Valores da mediana (Md), mínimo (Min) e máximo (Max) dos escores de avaliação do preenchimento por contraste nas veias digitais dos membros de bovinos $(\mathrm{P}<0,05)$

\begin{tabular}{cccccccc}
\hline Grupo & \multirow{7}{c}{$\begin{array}{c}\text { Escore de } \\
\text { preenchimento }\end{array}$} & \multicolumn{7}{c}{ Momentos } \\
\cline { 3 - 8 } & & 20 & 40 & 60 & 80 & 100 & 120 \\
\hline 1 & Md & 2 & 2 & 2 & 2 & 1 & 2 \\
$(\mathrm{n}=24)$ & Min & 0 & 0 & 0 & 0 & 0 & 0 \\
& Max & 4 & 4 & 4 & 4 & 4 & 4 \\
\hline 2 & Md & 3 & 3 & 3 & 3 & 3 & 3 \\
$(\mathrm{n}=29)$ & Min & 0 & 0 & 0 & 0 & 0 & 0 \\
& Max & 5 & 5 & 5 & 5 & 5 & 5 \\
\hline & $\mathrm{P}^{1}$ & 0,013 & 0,008 & 0,008 & 0,0113 & 0,006 & 0,013 \\
\hline
\end{tabular}

Para a variável grau de radiopacidade do contraste na venografia, não existe diferença significativa $(\mathrm{P}>0,05)$ quando se comparam os momentos, acessos e grupos (Tab. 2).

Rucker (2007) recomenda que as radiografias sejam tomadas no período imediatamente após a administração do contraste. Em outros estudos, sugere-se que sejam tomadas entre 45 e 120 segundos, sendo este último utilizado para diagnóstico de abscessos, seroma ou edema
(Rucker, 2007; Rucker, 2010; D’Arpe e Bernardini, 2010).

Neste estudo, não foram observadas diferenças significativas entre os momentos M1 e M6, quando se avaliam os padrões de preenchimento vascular e radiopacidade do contraste, diferentemente do observado em ovinos $\mathrm{e}$ caprinos, em que a difusão completa do contraste, de forma a caracterizar o momento ideal para a tomada de imagens, ocorreu aos 60 segundos (Santos et al.,2015a).

Tabela 2. Valores da mediana (Md), mínimo (Min) e máximo (Max) dos escores de avaliação da radiopacidade do contraste nas veias digitais dos membros de bovinos $(\mathrm{P}<0,05)$

\begin{tabular}{cccccccc}
\hline Grupo & \multirow{7}{*}{$\begin{array}{c}\text { Escore de } \\
\text { radiopacidade }\end{array}$} & \multicolumn{7}{c}{ Momentos } \\
\cline { 3 - 8 } & & 20 & 40 & 60 & 80 & 100 & 120 \\
\hline 1 & Md & 3 & 3 & 3 & 3 & 3 & 3 \\
$(\mathrm{n}=24)$ & Min & 1 & 1 & 1 & 1 & 1 & 1 \\
& Max & 3 & 3 & 3 & 3 & 3 & 3 \\
\hline 2 & Md & 3 & 3 & 3 & 3 & 3 & 3 \\
$(\mathrm{n}=29)$ & Min & 2 & 2 & 2 & 1 & 1 & 1 \\
& Max & 3 & 3 & 3 & 3 & 3 & 3 \\
\hline & $\mathrm{P}^{1}$ & 0,153 & 0,153 & 0,286 & 0,453 & 0,722 & 0,722 \\
\hline
\end{tabular}

\section{CONCLUSÃO}

A administração de $20 \mathrm{~mL}$ de contraste (diatrizoato de meglumina) apresentou melhor preenchimento vascular e radiopacidade. Não houve diferença no preenchimento vascular e na radiopacidade entre 20 e 120 segundos, após a administração do contraste. Os diferentes acessos venosos praticados: digital dorsal comum III, digital palmar/plantar comum II e digital palmar/plantar comum IV, não influenciaram na venografia. 


\section{REFERÊNCIAS}

ACKERMAN, N.; GARNER, H.E.; COFFMAN, J.R.; CLEMENT, J.W. Angiographic appearance of the normal equine foot and alterations in chronic laminitis. J. Am. Vet. Med. Assoc., v.166, p.58-62, 1975.

BALDWIN, G.; POLLIT, C.C. Progression of venographic changes after experimentally induced laminitis. Vet. Clin. N. Am. Equine. Pract., v.26, p.135-140, 2010.

BUDRAS, K.D.; SACK, W.O.; ROCK, S. Anatomy of the horse. London: Mosby-Wolfe, 2005. 199p.

D'ARPE, L.; BERNADINI, D. Digital venography in horse and its clinical application in Europe. Vet. Clin. N. Am. Equine Pract., v.26, p.339-359, 2010.

HOOD, D.M. The pathophysiology of developmental and acute laminitis. Vet. Clin. N. Am. Equine. Pract., v.15, p.321-43, 1999.

KÖNIG, H.E.; LIEBICH, H. Anatomia dos animais domésticos. 4.ed. Porto Alegre: Artmed, 2011. 834p.
MOORE, K.L. et al. Anatomia orientada para a clínica. Rio de Janeiro: Guanabara Koogan, 2014. 1136p.

REDDEN, R.F. A technique for performing digital venography in the standing horse. Equine. Vet. Educ., v.3, p.128-172, 2001

RUCKER A. The digital venogram. In: FLOYD, A.E. Equine podiatry. Philadelphia: WB Saunders, 2007. 328-346p.

RUCKER, A. Clinical applications of digital venography. J. Equine Vet. Sci., v.30, p.491-502, 2010.

SANTOS, I.F.C.; HUSSNI, C.A.; RODRIGUES, C.A.; WATANABE, M.J. et al. Técnica venográfica contrastada in vivo dos dígitos de ovinos e caprinos. Arq. Bras. Med. Vet. Zootec., v.67, p.1630-1638, 2015a

SANTOS, I.F.C.; HUSSNI, C.A.; RODRIGUES, C.A.; WATANABE, M.J. et al. Digital venography in ruminants - a review. Vet. Quarterly, v.36, p.22-29, 2015b.

STATISTICAL analysis system.Version 9.3. Cary: SAS Institute, 2008.

TORTORA, G.J.; DERRICKSON, B. Corpo humano: fundamentos de anatomia e fisiologia. Porto Alegre: Artmed, 2012. 398-404p. 